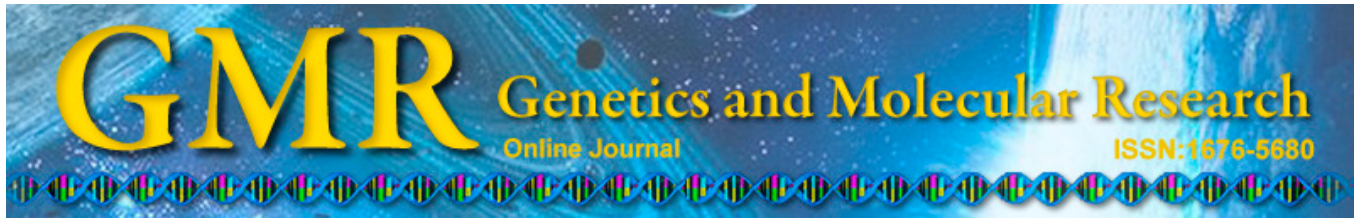

\title{
Development of microsatellite markers for Hancornia speciosa Gomes (Apocynaceae)
}

\author{
A.J.L. Rodrigues ${ }^{1}$, A.T. Yamaguishi ${ }^{2}$, L.J. Chaves ${ }^{3}$, A.S.G. Coelho ${ }^{3}$, \\ J.S. Lima ${ }^{4,5}$ and M.P.C. Telles ${ }^{4}$ \\ ${ }^{1}$ Universidade Estadual de Goiás, Anápolis, GO, Brasil \\ ${ }^{2}$ Embrapa - Recursos Genéticos e Biotecnologia, Brasília, DF, Brasil \\ ${ }^{3}$ Escola de Agronomia, Universidade Federal de Goiás, Goiânia, GO, Brasil \\ ${ }^{4}$ Instituto de Ciências Biológicas, Universidade Federal de Goiás, \\ Goiânia, GO, Brasil \\ ${ }^{5}$ Programa de Pós-Graduação em Ecologia e Evolução, \\ Universidade Federal de Goiás, Goiânia, GO, Brasil \\ Corresponding author: M.P.C. Telles \\ E-mail: tellesmpc@gmail.com
}

Genet. Mol. Res. 14 (3): 7274-7278 (2015)

Received June 6, 2014

Accepted February 20, 2015

Published July 3, 2015

DOI http://dx.doi.org/10.4238/2015.July.3.2

\begin{abstract}
Herein, we describe 34 microsatellite loci developed using an enrichment genomic library for the tree species Hancornia speciosa Gomes (Apocynaceae). Thirty-five individuals were genotyped using 34 primers to analyze the polymorphisms at each locus. The number of alleles per locus ranged from 4 to 20 . The average number of alleles was 8.11 , and the expected heterozygosity ranged from 0.62 to 0.94 . These microsatellite primers will be useful in population genetics studies for this species.
\end{abstract}

Key words: Cerrado; Enrichment genomic library; Mangaba; SSR; STR 


\section{INTRODUCTION}

Microsatellites are noncoding, repetitive DNA regions that consist of tandemly repeated small motifs. These markers have been an important tool in population genetic studies of natural species due to their reproducibility, multiallelic nature, codominant inheritance, relative abundance, and wide genome coverage (Morgante and Olivieri, 1993; Goldstein and Schlotterer, 1999). Nevertheless, the use of microsatellite loci in neotropical tree species is still scarce, in part due to the difficulties in developing specific markers and transferring them from closely related species (Telles et al., 2011).

Hancornia speciosa Gomes (Apocynaceae) is a widely distributed Neotropical plant species native to Brazil; it occurs in the north, northeast, and central regions of the country, mainly in the Cerrado biome. Its fruits possess agroindustrial potential and stand out for the taste of their derivative products, which are highly appreciated and, thus, easily marketable (Nogueira and Albuquerque, 2006; Silva-Júnior et al., 2006).

Knowledge of the genetic structure of this species may provide important information for developing conservation and management programs, but microsatellite markers are not available for this species. Because the species is monotypic within the genus, the possibility of transferability is potentially low, and the best current technological strategy to deal with lack of markers is to specifically develop them using an enriched genomic library. Thus, the aim of this study was the development of microsatellite loci for this species.

\section{MATERIAL AND METHODS}

A microsatellite-enriched library was constructed using total genomic DNA of one individual of $H$. speciosa based on the protocol developed by Rafalski et al. (1996). The genomic DNA was extracted from expanded leaves following the standard 2\% CTAB procedure (Doyle and Doyle, 1990); it was then digested by the restriction enzymes Sal3AI (GATC), MseI (TAA), and TspI 509 (AATT). Digested fragments ranging in size between 200 and 800 bp were gel-extracted using the Qiaquick Gel Extraction Kit (Qiagen). The DNA fragments were linked for $12 \mathrm{~h}$ at $12^{\circ} \mathrm{C}$ to the adaptors $T s p \mathrm{I}$, prepared from long (5'-AATTGGAATTCGACTCGCAGCAGCC-3') and short (5'-GGCTGCTGCGAGTCGAATTCC-3') oligos in a reaction including 3 U T4 DNA ligase (Amersham Pharmacia Biotech). The amplified DNA fragments were hybridized with a probe (TC) $)_{13}$ linked to biotin. The hybridization mixture was captured by coated magnetic beads (Dynabeads, Streptavidina Bohering Mannhein). The enriched DNA obtained was amplified through 20 cycles using primers complementary to the adaptor sequence. The purified DNA was cloned into a pGEM-T Easy Vector (Promega Corp.), and plasmids were inserted into transformed Escherichia coli competent cells. The positive clones were amplified through polymerase chain reaction (PCR) using the M13 primers (forward and reverse) and sequenced on an ABI 3000 semi-automated sequencer.

The regions containing microsatellites were analyzed according to the quality of the flanking regions and repetitive sequences. Primer pairs for these regions were designed with the Primer3 software (Rozen and Skaletsky, 2000). The Tandem Repeats Finder software (Benson, 1999) was used to identify microsatellite regions with length $\geq 18 \mathrm{bp}$. The quality of the sequences was analyzed with the Phred software (Ewing et al., 1998) and aligned with the BioEdit 5.0.0 (Hall, 1999) and Clustal X (Thompson et al., 1997) software programs. 
Thirty-five individuals of $H$. speciosa from different populations were genotyped to characterize the polymorphism at the developed loci. Microsatellite amplifications were performed in $25 \mu \mathrm{L}$ volume reactions containing $10 \mathrm{ng}$ DNA, 1 X reaction buffer, $1.5 \mathrm{mM} \mathrm{MgCl}_{2}$, $0.4 \mathrm{mM}$ dNTP, $0.1 \mu \mathrm{M}$ primer, and $1 \mathrm{U}$ Taq DNA polymerase. PCRs were carried out in a PTC-100 thermal cycler (MJ Research Inc.) with the following conditions: $94^{\circ} \mathrm{C}$ for $5 \mathrm{~min}$ (one cycle), $94^{\circ} \mathrm{C}$ for $1 \mathrm{~min}, 48^{\circ}$ to $64^{\circ} \mathrm{C}$ (according to each primer annealing temperature, see Table 1 ) for $1 \mathrm{~min}, 72^{\circ} \mathrm{C}$ for $1 \mathrm{~min}\left(30\right.$ cycles), and $72^{\circ} \mathrm{C}$ for $7 \mathrm{~min}$ (one cycle). Amplified products were separated in $6 \%$ denaturing polyacrylamide gels stained with silver nitrate (Creste et al., 2001) and sized by comparison with 10- and 100-bp DNA ladder standards (Invitrogen, Frederick, MD, USA).

The number of alleles per locus $\left(N_{\mathrm{A}}\right)$ and the observed $\left(H_{\mathrm{O}}\right)$ and expected heterozygosities $\left(H_{\mathrm{E}}\right)$ under Hardy-Weinberg equilibrium $(\mathrm{Nei}, 1978)$ were estimated with the GDA software (Lewis and Zaykin, 2001).

Table 1. Characterization of the 34 microsatellite loci developed for Hancornia speciosa, based on 35 individuals.

\begin{tabular}{|c|c|c|c|c|c|c|c|}
\hline Locus & Repeat motif & Primer sequence $\left(5^{\prime}-3^{\prime}\right)$ & Expected size (bp) & Allele range (bp) & $\mathrm{Ta}\left({ }^{\circ} \mathrm{C}\right)$ & $N_{\mathrm{A}}$ & $H_{\mathrm{E}}$ \\
\hline HS 01 & $(\mathrm{GCA})_{6}(\mathrm{TC})_{20}(\mathrm{GCA})_{8}$ & $\begin{array}{l}\text { F: GTGTCTTCCATCCGAGCTTAAC } \\
\text { R: TTTCCCAGAAAGGAGAGGTACA }\end{array}$ & 275 & $250-310$ & 50 & 13 & 0.89 \\
\hline HS 02 & $(\mathrm{CT})_{14}$ & $\begin{array}{l}\text { F: AATTCAACCCTTCTGCGAATC } \\
\text { R: CACCAGGAACGATCAGGAAG }\end{array}$ & 169 & $80-150$ & 50 & 4 & 0.68 \\
\hline HS 03 & $(\mathrm{CT})_{5}(\mathrm{CT})_{6}$ & $\begin{array}{l}\text { F: CACTCTCTCTCAGCATTTCCCT } \\
\text { R: TGCATAGAAGGAGAAGAAGAAGC }\end{array}$ & 158 & $120-180$ & 58 & 7 & 0.81 \\
\hline HS 04 & $(\mathrm{AG})_{17}$ & $\begin{array}{l}\text { F: GCGAGTCGAATTCCAATTACTC } \\
\text { R: AAAGTCCAGTAATAGCGCCAAA }\end{array}$ & 247 & $80-200$ & 54 & 13 & 0.84 \\
\hline HS 05 & $(\mathrm{GA})_{15}(\mathrm{TGC})_{6}$ & $\begin{array}{l}\text { F: GGGTGTACTGCCACAAGGTACT } \\
\text { R: GTGTCTTCCATCCGAGCTTAAC }\end{array}$ & 177 & $200-300$ & 58 & 7 & 0.73 \\
\hline HS 06 & $(\mathrm{GA})_{14}$ & $\begin{array}{l}\text { F: CGGCTGTATTAAGTCTATTGCCA } \\
\text { R: CCCTGCCACTCTCTTTTCC }\end{array}$ & 107 & $100-150$ & 50 & 7 & 0.75 \\
\hline HS 07 & $(\mathrm{CT})_{6}(\mathrm{TC})_{5}(\mathrm{CA})_{6}$ & $\begin{array}{l}\text { F: ACTCACACAAAGCTCACACACC } \\
\text { R: AGGTAATCAGTTCTGGGGAGGT }\end{array}$ & 261 & $220-260$ & 56 & 5 & 0.69 \\
\hline HS 08 & $(\mathrm{CA})_{6}(\mathrm{CT})_{17}$ & $\begin{array}{l}\text { F: AATGTAGAGGTGAACGAGTGGG } \\
\text { R: TACACCCTGCTCATCGTTTATG }\end{array}$ & 248 & $200-250$ & 48 & 12 & 0.89 \\
\hline HS 09 & $(\mathrm{CT})_{7}(\mathrm{CT})_{10}(\mathrm{CT})_{6}$ & $\begin{array}{l}\text { F: TGCAAACCCTCGTTTATTTCTT } \\
\text { R: ATTGTGTGTGTTGTGTGTGGG }\end{array}$ & 262 & $200-300$ & 64 & 8 & 0.81 \\
\hline HS 10 & $(\mathrm{CT})_{14}(\mathrm{CT})_{8}$ & $\begin{array}{l}\text { F: ACAAATCAATGAGGAGGTGCTT } \\
\text { R: TAACTATGTGCAACCGCAAGAC }\end{array}$ & 135 & $100-200$ & 52 & 8 & 0.85 \\
\hline HS 11 & $(\mathrm{GA})_{17}$ & $\begin{array}{l}\text { F: GTGATATTTCGTGCTCTCCAAG } \\
\text { R: CTCTGCCACTGTGCAACC }\end{array}$ & 106 & $100-200$ & 50 & 8 & 0.77 \\
\hline HS 12 & $(\mathrm{CT})_{22}$ & $\begin{array}{l}\text { F: CAAACCCTCGTTCTTCTCTTCT } \\
\text { R: GATGTCGCAACTCGAGCA }\end{array}$ & 214 & $200-250$ & 56 & 5 & 0.66 \\
\hline HS 13 & $(\mathrm{CT})_{12}$ & $\begin{array}{l}\text { F: CTGGGGTACTTCAGCAAATCAC } \\
\text { R: CATCAAAGACCGTTGTCTCCTT }\end{array}$ & 101 & $100-150$ & 56 & 8 & 0.84 \\
\hline HS 14 & $(\mathrm{TC})_{14}$ & $\begin{array}{l}\text { F: GAGCAGGAGTCAGGAAAATCAC } \\
\text { R: ACAGTGAAGGGGCAATGAAG }\end{array}$ & 126 & $100-200$ & 56 & 6 & 0.71 \\
\hline HS 15 & $(\mathrm{GA})_{16}$ & $\begin{array}{l}\text { F: GTGAGTGTTGTGCGTGTGTGT } \\
\text { R: CTTCTTCTTCCTCTCGCGGT }\end{array}$ & 116 & $150-300$ & 60 & 5 & 0.62 \\
\hline HS 16 & $(\mathrm{GA})_{12}$ & $\begin{array}{l}\text { F: CGTTGGTAGCGGCTGTATTAAG } \\
\text { R: CCCCTCCTGCCACTCTCT }\end{array}$ & 114 & $100-150$ & 48 & 7 & 0.72 \\
\hline HS 17 & $(\mathrm{GA})_{16}$ & $\begin{array}{l}\text { F: ACTCGAGCAGAAGAAGCAAATC } \\
\text { R: ACACACCCTCATCAGCCC }\end{array}$ & 116 & $100-200$ & 54 & 11 & 0.82 \\
\hline HS 18 & $(\mathrm{AG})_{14}$ & $\begin{array}{l}\text { F: ATTCATGCTCCACTGGCTTC } \\
\text { R: GACCACAGCTAGTGACGTGTTC }\end{array}$ & 207 & $300-350$ & 50 & 11 & 0.87 \\
\hline HS 19 & $(\mathrm{TTC})_{5}(\mathrm{GCC})_{6}$ & $\begin{array}{l}\text { F: ATTCTGCAAACCCTCATTTC } \\
\text { R: AAAGAGAGAGTGTGTGTGCG }\end{array}$ & 109 & $350-400$ & 56 & 4 & 0.63 \\
\hline HS 20 & $(\mathrm{CT})_{11}$ & $\begin{array}{l}\text { F: CTAACCCACTACAACTCTGGGC } \\
\text { R: ATCCGATTTAGGACATTGGGT }\end{array}$ & 140 & $200-250$ & 50 & 9 & 0.84 \\
\hline
\end{tabular}

Continued on next page 


\begin{tabular}{|c|c|c|c|c|c|c|c|}
\hline Locus & Repeat motif & Primer sequence $\left(5^{\prime}-3^{\prime}\right)$ & Expected size (bp) & Allele range (bp) & $\mathrm{Ta}\left({ }^{\circ} \mathrm{C}\right)$ & $N_{\mathrm{A}}$ & $H_{\mathrm{E}}$ \\
\hline HS 21 & $(\mathrm{AG})_{13}$ & $\begin{array}{l}\text { F: GATTGTGTGTGGTGTGTGTGG } \\
\text { R: CCCTCGTTCTTCTCTTCTTCTTC }\end{array}$ & 207 & $200-300$ & 64 & 7 & 0.76 \\
\hline HS 22 & $(\mathrm{GA})_{12}$ & $\begin{array}{l}\text { F: GGACGAAACGAAATGGAGAGTA } \\
\text { R: AGTAAAGACACGTCATCCCCAC }\end{array}$ & 160 & $180-220$ & 56 & 7 & 0.74 \\
\hline HS 23 & $(\mathrm{CGC})_{6}$ & $\begin{array}{l}\text { F: TGCAAACCCTCATTTCTTTTCTTC } \\
\text { R: GGAGCAAATCGGGAAGCC }\end{array}$ & 134 & $180-250$ & 56 & 9 & 0.84 \\
\hline HS 24 & $(\mathrm{AG})_{12}$ & $\begin{array}{l}\text { F: GCTAAATCAAGCAAACCTCGAC } \\
\text { R: AAAGCAGTCCATGATCCATTTC }\end{array}$ & 152 & $200-300$ & 58 & 7 & 0.81 \\
\hline HS 25 & $(\mathrm{TC})_{11}$ & $\begin{array}{l}\text { F: CACGGCTGTCTGTCGTTG } \\
\text { R: ACTCGAGCAGAAGAAGCAAATC }\end{array}$ & 118 & $80-150$ & 56 & 6 & 0.65 \\
\hline HS 26 & $(\mathrm{CT})_{10}$ & $\begin{array}{l}\text { F: CAAACAAGCTTTATGTGGGTCA } \\
\text { R: AGCTCAAGGAAGTGGGATCTAA }\end{array}$ & 172 & $200-300$ & 58 & 9 & 0.80 \\
\hline HS 27 & $(\mathrm{GA})_{14}$ & $\begin{array}{l}\text { F: TATAGTGGTCCTGCACCCTTGT } \\
\text { R: TTTTCCCTTGTGCTTCGC }\end{array}$ & 128 & $100-150$ & 54 & 20 & 0.94 \\
\hline HS 28 & $(\mathrm{CT})_{15}$ & $\begin{array}{l}\text { F: TCTATTGGAGTGTTTCCCTCAC } \\
\text { R: AGATTCAAGAGAAAACGGAAAA }\end{array}$ & 129 & $250-300$ & 56 & 5 & 0.76 \\
\hline HS 29 & $(\mathrm{CT})_{15}$ & $\begin{array}{l}\text { F: GTGAGCTTGTGCTACTCTCCCT } \\
\text { R: TTGTCACCGTGTTTCTAGCAGT }\end{array}$ & 178 & $300-400$ & 50 & 6 & 0.68 \\
\hline HS 30 & $(\mathrm{AG})_{10}$ & $\begin{array}{l}\text { F: GAGGAATCTCAGCCAAGTCCTA } \\
\text { R: CCCAGCCTCTACAAACTCTCTG }\end{array}$ & 169 & $180-200$ & 56 & 10 & 0.88 \\
\hline HS 31 & $(\mathrm{AG})_{19}$ & $\begin{array}{l}\text { F: GTCGATCCGATTTAGGACATTG } \\
\text { R: GCACACACCCACACTCCA }\end{array}$ & 176 & $50-100$ & 56 & 5 & 0.70 \\
\hline HS 32 & $(\mathrm{CT})_{9}$ & $\begin{array}{l}\text { F: CTTTTCTGCAACCCTCATTCTT } \\
\text { R: ACTCGAGCTGAGAAAACGAATC }\end{array}$ & 185 & $200-300$ & 52 & 14 & 0.90 \\
\hline HS 33 & $(\mathrm{AG})_{24}$ & $\begin{array}{l}\text { F: CGTTGGTAGCGGCTGTATTAAG } \\
\text { R: CACTCTCTTTTCCCGATTTTCC }\end{array}$ & 132 & $80-120$ & 56 & 9 & 0.83 \\
\hline HS 34 & $(\mathrm{TGG})_{5}$ & $\begin{array}{l}\text { F: GCATCTTGAGAAGAAAGAGGGA } \\
\text { R: TGTAGATGGAATCTTGCCACTG }\end{array}$ & 144 & $180-200$ & 60 & 4 & 0.65 \\
\hline Average & - & - & - & - & - & 8.11 & 0.77 \\
\hline
\end{tabular}

Ta = annealing temperature; $N_{\mathrm{A}}=$ number of alleles; $H_{\mathrm{E}}=$ expected heterozygosity.

\section{RESULTS AND DISCUSSION}

From the 1056 positive clones, we sequenced a total of 576. It was possible to design primers in the conservative region for 74 sequences with different compositions, sizes, and repeat motifs. Dinucleotide regions, which typically exhibit higher polymorphism than that of other regions (Chakraborty et al., 1997), were preferentially chosen for amplification. From these 74 microsatellite loci, 34 were selected for synthesis and amplification (Table 1). The selection of some trinucleotide regions occurred in an attempt to obtain greater coverage of the genome.

All loci presented polymorphisms, with the $N_{\mathrm{A}}$ per locus ranging from 4 to 20. Average allelic richness was 8.11 and the $H_{\mathrm{E}}$ was high, ranging from 0.62 to 0.94 (Table 1). Thus, the microsatellites developed herein revealed high genetic diversity in the species studied and may allow individual discrimination, with many potential applications for population studies (i.e., pollen dispersal, population structure, and gene flow). Based on the above parameters, we conclude that the set of molecular markers developed herein is adequate for further use in population genetics studies for this species, allowing both the understanding of microevolutionary processes and the establishment of more effective conservation strategies for this species.

\section{REFERENCES}

Benson G (1999). Tandem repeats finder: a program to analyze DNA sequences. Nucleic Acids Res. 19: 573-580.

Chakraborty R, Kimmel M, Stivers DN, Davison LJ, et al. (1997). Relative mutation rates at di-, tri-, and tetranucleotide microsatellite loci. Proc. Nat. Acad. Sci. U. S. A. 94: 1041-1046. 
Creste S, Tulmann Neto A and Figueira A (2001). Detection of single sequence repeat polymorphisms in denaturing polyacrylamide sequencing gels by silver staining. Plant Mol. Biol. Rep. 19: 299-306.

Doyle JJ and Doyle JL (1990). Isolation of plant DNA from fresh tissue. Focus 12: 13-15.

Ewing B, Hillier L, Wendl MC and Green P (1998). Base-calling of automated sequencer traces using phred. I. Accuracy assessment. Genome Res. 8: 175-185.

Goldstein DB and Schlotterer C (1999). Microsatellites: evolution and applications. Oxford University Press, New York.

Hall TA (1999). BioEdit: a user-friendly biological sequence alignment editor and analysis program for Windows 95/98/ NT. Nucleic Acids Symp. Ser. 41: 95-98.

Lewis PO and Zaykin D (2001). Genetic Data Analysis: computer program for the analysis of allelic data. Version 1.0 (d16c). Available at [http://www.eeb.uconn.edu/people/plewis/software.php].

Morgante M and Olivieri AM (1993). PCR-amplified microsatellites as markers in plant genetics. Plant J. 3: 175-182.

Nei M (1978). Estimation of average heterozygosity and genetic distance from a small number of individuals. Genetics 89: 583-590.

Nogueira RJMC and Alburquerque MB (2006). Ecofisiologia. In: A cultura da Mangaba (Silva-Júnior JF and Lédo AS, eds.). 1st edn. Embrapa Tabuleiros Costeiros, Brasília, 36-42.

Rafalski JA, Vogel JM, Morgante M, Powell W, et al. (1996). Generating and using DNA markers in plants. In: Non mammalian genomic analysis: a practical guide. Academic Press, New York, 75-134.

Rozen S and Skaletsky HJ (2000). Primer3: bioinformatics methods and protocols. In: Methods in Molecular Biology (Krawetz S and Misener S, eds.). Humana Press, Totowa, 365-386.

Silva Júnior JF, Araújo IA, Barreira Neto M, Espíndola ACM, et al. (2006). Recursos genéticos nos tabuleiros costeiros e baixada litorânea do Nordeste. In: A cultura da Mangaba (Silva-Júnior JF and Lédo AS, eds.). 1st edn. Embrapa Tabuleiros Costeiros, Brasília, 58-72.

Telles MPC, Peixoto FP, Lima JS, Resende LV, et al. (2011). Development of microsatellite markers for the endangered Neotropical tree species Tibouchina papyrus (Melastomataceae). Genet. Mol. Res. 10: 321-325.

Thompson JD, Gibson TJ, Plewniak F, Jeanmougin F, et al. (1997). The CLUSTAL_X windows interface: flexible strategies for multiple sequence alignment aided by quality analysis tools. Nucleic Acids Res. 25: 4876-4882. 